\title{
The design and implementation of satellite communication signal spectrum automatic monitoring System
}

\author{
Liu Mingbo \\ China Satellite Maritime Tracking and Control Department \\ Jiangyin, China \\ mingbo2006@163.com
}

\author{
Wang Zhong \\ China Satellite Maritime Tracking and Control Department \\ Jiangyin, China \\ wZhong@163.com
}

\author{
Chang Jishi \\ China Satellite Maritime Tracking and Control Department \\ Jiangyin, China \\ cjShi@163.com
}

\begin{abstract}
This article introduces the design idea and implement process of satellite communications signal Auto-Monitor System based on GPIB interface, and puts forward the system design frame centered with spectrum analyzer of "frequency spectrograph + GPIB/USB converter+ computer", detailed introduces the implement method of Auto-Monitor application system. Tests show that system works stably, has good Real-Time property and high data precision. The system satisfies the requirement and is provided with some popularization and applied value.
\end{abstract}

Keywords — satellite communication; automatic monitoring System; General Purpose Interface Bus; LabWindows/CVI.

I. Introduction

With the development of electronic technology, communication technology and computer technology, all kinds of automatic monitoring technology has also got rapid development, automatic monitoring system which is under the computer's control will make the monitoring process become efficient, simple and flexible[1]. In the management and applications of satellite station, the real-time monitoring of RF signal spectrum mainly adopts the way that frequency spectrograph directly measures the signal spectrum and the personnel artificially observes the real-time spectrum distribution to determine the working condition of satellite communications equipment. Because there is no automatic monitoring function, the monitoring and management about spectrum mainly uses artificial method which has low efficiency. If we can make full use of the frequency spectrograph's input/output interface to achieve the automatic monitoring functions of automatic extracting, recording, analysis and abnormal alarming, the monitoring efficiency and cognition degree of satellite communication system will be significantly improved.

\section{The overall design}

GPIB (General Purpose Interface Bus) is a communication protocol between automatic testing equipments [2]. With the development and popularization of GPIB interface system and the electronic computer, almost all major equipments contain ready-made GPIB interface which provides ways for customers to realize remote automatic monitoring function, therefore, our automatic monitoring system using the existing GPIB interface provided by frequency spectrograph to implement the 
Real-Time automatic monitoring of satellite communications signal spectrum, which includes Real-Time achieving monitoring data, automatic signal analysis, processing and abnormal alarm. Its main function has the following points:

(1) The monitoring and early warning of RF signal data

At present, the state management of Satcom stations is mainly based on the parameter monitoring on the up/down transducer and modem to achieve the monitoring function of intermediate frequency signal input/output state. The monitoring of RF signal mainly uses the frequency spectrograph, which does not have automatic monitoring function, but offers GPIB interface. Our system is designed to get spectrometer data through the GPIB interface, and achieve the automatic monitoring function of RF signal. Once the uplink or downlink signal appears the fluctuation or loss phenomenon of carrier signal, the monitoring system will detect them in time and send out alarm.

(2) The recording and analysis of RF signal

Via GPIB interface, the monitoring computer regularly sends commands to the spectrometer to continue to get the spectrum information and establish the RF signal database, which realized the real-time acquisition, archiving, and quantitative analysis of RF signal. On the edge of the communication satellite beam coverage area, signal level fluctuation is relatively frequent, it is necessary to accurately and long-term records of signal level for the timely acquiring the state of Satcom

(3) The spectrum management

We can establish the frequency resource database based on the current communication frequency resource, take analysis and comparison with the real-time spectrum occupancy data which is automatically obtained, and use the visual interface to achieve the simple management. It is a good solution for the current communication frequency spectrum occupancy and the spectrum sharing problem to intuitively understand the spectrum usage situation and prevent negative consequences, such as, spectrum usage conflict and communication interferer.

The System is implemented mainly based on the GPIB interface of spectrometer (take Agilent E4408B for example), and complete the function of the spectrometer data and analysis results real-time acquiring, analysis, recording, displaying, fault alarm, etc. The overall design structure is shown in Figure 1.

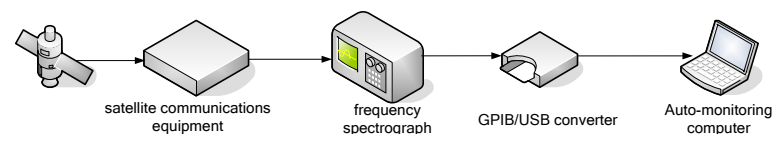

Fig 1. The system overall design structure

It can be seen from figure 1 that the monitoring system consists of hardware and software. Among them, the system hardware consists of spectrometer, GPIB-USB converter and monitoring computer. Spectrometer is mainly used to complete Satcom RF signal's real-time acquisition, frequency domain analysis, numerical calculation and real-time display function, and provide GPIB interface; GPIB-USB converter mainly provides data transmission channel between spectrometer and monitoring computer; Monitoring computer provides the platform for system applications which achieves the functions of monitoring data real-time acquiring, analysis, recording, displaying and fault alarming, etc

The GPIB-USB converter adopts Agilent 82357B USB/GPIB Interface, which achieves the converting and data transmission between GPIB and USB2.0 [3].

System application is the core of the entire automatic monitoring system, which is designed to work in Microsoft Windows/XP operating system, be provided with a flexible operation mode and friendly man-machine interface. According to the system function, the system application is further divided into driver, data communication, real-time record storage, real-time displaying, and abnormal alarming five function modules. The composing of system application is shown in Figure 2. 


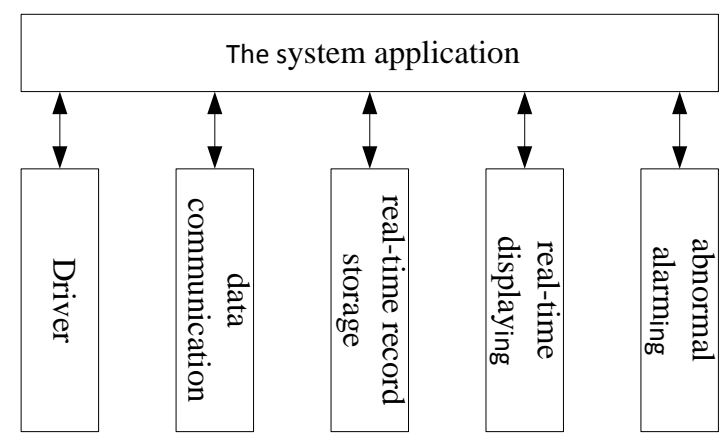

Fig 2. The composing of system application

System application is complemented basing on LabWindows/CVI and Visual $\mathrm{C}++$ language, NI LabWindows/CVI is specialized in the design of virtual instrument software, which contains rich resources of interface design and function library, possesses the advantages of convenient use and flexible design[4]. But the Agilent 82357B USB/GPIB Interface converter only provides the C-based drive function library, which can't be called directly by LabWindows/CVI software, therefore, system using Visual $\mathrm{C}++$ language to make secondary packaging of the drive function library, and provide calling Interface for LabWindows/CVI.

\section{System implementation}

System mainly realizes the functions on Agilent E4408B Spectrum Analyzer of Real-time parameter setup and data acquisition, analysis, recording, display, alarming when falling across abnormal data. The hardware of the system is mainly achieved by adopting the industry mature product to make simple interconnection. Therefore, here we mainly refer to the implementation of the system application.

(1) The design of driver program

As mentioned earlier, the Agilent 82357B USB/GPIB Interface convertor only support the $\mathrm{C}$ language-based driver library, the system uses the technique of Visual $\mathrm{C}++$ dynamic link library (DLL) to make second package about the GPIB driver function library, which provides interfaces for calling by LabWindows / CVI. Only the exported functions in the DLL can be called through the dynamic link library by other programs. There are two ways for exporting functions from the DLL[5]:

- Using Module-definition file
Module-definition file is a text file which is composed of one or more statements describing the DLL attribute. It lists the name and serial name of exported functions after the EXPORTS statement. If NONAME flag is put after the function name, it indicates that system only export the serial number of the output function, the application can only use the serial number to access the DLL exported functions. This way has a certain security, and save memory space for storing the function name string.

- Using the keyword _ declspec (dllexport)

We use _declspec (dllexport) keyword in the function definition, then, the function, class and class member functions can be exported from the DLL. If you use this keyword, you do not need to write DEF file. The system automatically generates LIB and DLL files for the application in the process of compilation. This method can easily export functions. Therefore, the completion of exporting the interface functions only need to use the declaration _ declspec (dllexport), then compile and link the functions.

The DLL package library which is used in this system is generated by the second method. As follows are parts of the interface functions:

extern "C" _declspec(dllexport) signed long CVIviPrintf(unsigned long vi,char *writeFmt); // Set command parameters to the spectrum analyzer

extern "C" _declspec(dllexport) signed long CVIviOpen(unsigned long *defaultRM,unsigned long *vi); //Open the GPIB interface device

extern "C" _declspec(dllexport) signed long CVIviQueryf(unsigned long vi,char *writeFmt,char *str,int *val); //Obtain data from devices

extern "C" _declspec(dllexport) signed long CVIviQueryfASCII(unsigned long vi,int *num,char *cResult); // Obtain ASCII format waveform data from the spectrum analyzer

(2) The design of Data communication

Same with other protocols, GPIB protocol is according to a certain data format to process data transmission, therefore, the data communication process is carried out in accordance with a the GPIB prearranged data format. On the 
basis of finishing the driver function design, according to actual needs, we reference to the description of the command and data format on the Agilent E4408B Spectrum Analyzer Datasheet, send a certain order and format of the various types of commands to the spectrum analyzer, and then wait to receive the back data.

The Real-time acquiring of the spectrum analyzer waveform data uses LabWindows / CVI's asynchronous clock control (Asynchrony Timer) which adopts multi-threading technology and read a spectrum analyzer data every 0.2 seconds, and completes all kinds of the follow-up capabilities.

(3) The design of application interface

System uses LabWindows / CVI's rich interface design resources to complete the design of the main interface, use the rich library to complete the various types functions of data recording, storage, display and abnormal alarm, its runtime interface shown in Figure 3 below.

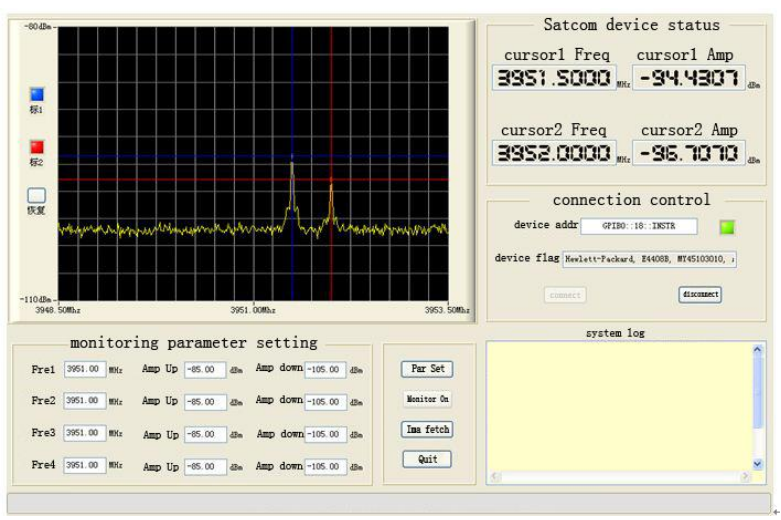

Fig 3. The runtime interface of system application

In Figure 3, "the connection control unit" completes the connection with spectrum analyzer and status display; "the graphical control" completes the functions of real-time spectrum analyzer waveform acquisition and display; "the Satcom device status unit" mainly completes the real-time display of the graphical controls cursor's frequency and amplitude value; "the monitoring parameter setting unit" complete the functions of setting the monitoring frequency and amplitude threshold vale. When the monitoring frequency spectrum amplitude exceeds the threshold limit of the allowable range, the data will trigger the abnormal alarm function to remind staff positions to deal by monitoring computer sound card to issue a voice alarm.

In addition, the system has a remote automatic setting function of the spectrum analyzer equipment commonly used parameters. The setup interface is shown in Figure 4.

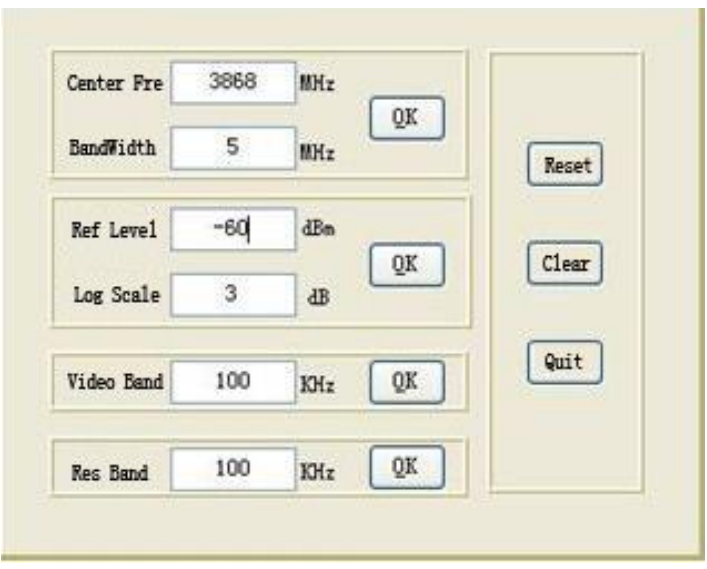

Fig 4. The remote automatic setting interface

In Figure 4, at the same time of setting the spectrum analyzer center frequency, bandwidth and reference level, logarithmic scale and other parameters, the $\mathrm{X}$-axis, $\mathrm{Y}$-axis scale of the graphical control in the main interface will update to ensure the monitoring computer interface consist with the real spectrum analyzer display interface.

The system has a friendly man-machine interface, completes the functions of E4408B spectrum analyzer parameter settings and data acquiring, analysis, recording, display and abnormal alarm and etc. Based on the analysis data, system can establish Satcom spectrum status information analysis database. In order to be able to intuitive and comprehensive understand the spectrum usage, our system records the spectrum data and waveform diagram every 10 minutes when the data is normal (data is in allowable threshold range), and records the spectrum data and waveform diagram every 0.2 seconds when the data is abnormal (data is out of allowable threshold range). In addition, the system also has the log recording function, which Real time records the various operations and data changes in the monitoring process. 
IV. Calculating and test of system index

Based on actual demand, here we mainly test the Real-time nature and accuracy characteristic of the system.

As the Agilent 82357B USB / GPIB Interface's typical nominal data transmission rate is not less than $1.15 \mathrm{MB} / \mathrm{S}$, and the spectrum analyzer waveform data amount is $401 *$ $13=5213$ Byte, therefore, the transmission time of the spectrum analyzer waveform data is only $5213 \mathrm{~B} \div 1.15 \mathrm{MB} / \mathrm{s}$ $=0.00432$ (second). System reads the spectrum data and refreshes waveform display every 0.2 seconds, therefore, the time delay between the waveforms displayed in the waveform monitor computer with the actual spectrum analyzer is 0.2 seconds. In the actual use process, abnormal carrier usually lasts more than 0.2 seconds, so the system index meets the actual demand.

The data accuracy test adopts the method of comparing the calibration frequency amplitude between obtained on the actual spectrum analyzer and displayed on the monitor computer. Selected calibration frequency $3.9515 \mathrm{GHz}$ in actual use of satellite communication equipment, the spectrum analyzer and monitoring computer display waveform is shown in Figure 5.
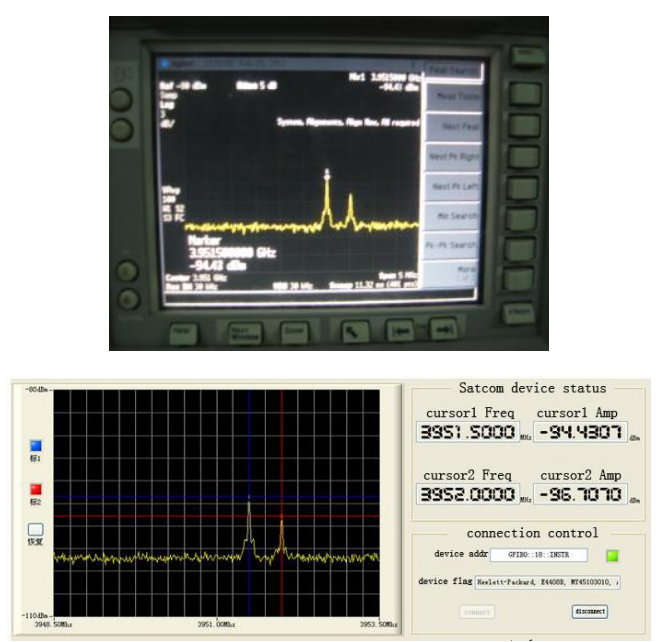

Fig 5. Comparing between the spectrum analyzer and monitoring computer

In Figure 5, the amplitude of $3.9515 \mathrm{GHz}$ frequency point displayed on spectrum analyzer is $-94.43 \mathrm{dBm}$, monitoring computer display is $-94.4307 \mathrm{dBm}$, the accuracy characteristic meets the actual demand.
V. Conclusion

In Satellite communication process, satellite communication station carrier spectrum monitoring is an important means to check both RF systems. Commonly used spectrum analyzer means of monitoring are only used for manual interpretation and monitoring, which cannot timely, accurately records flashed spectrum exception carrier. Our system has a good solution for above problems, further enhance the Real-time nature and accuracy characteristic of satellite communication station monitoring, and provides data, waveform real-time recording and abnormal data alarm function, can be extended to other signal automatic monitoring system, has a certain practical value.

[1] Feng Xin Liu. "Introduction to Computer Aided Test Technology (in chinese)“. Publishing House of Electronics Industry.(2004)

[2] Shi Qi Zhang. "Automatic Test System (in chinese)". University of Electronic Science \& Technology Press. (1990)

[3] Xi Chai. "Communication between Computers and test equipment with GP-IB interface(in chinese)“. Electrical Measurement \& Instrumentation. Vol.4. (2008)

[4] National Instruments Corporation. "Measurement Studio Reference“. National Instruments Corporation. (2001)

[5] Xue Feng Wang. "The Design of GPIB Interface Chip and Application in Logic Analyzer”. XiHua University. (2010) 